\section{A Practical Guide to Scientific Data Analysis}

by David Livingstone: Wiley, Chichester, UK, 2009, pp xv + 341, ISBN 978-0-47085153-1. Price: GBP 50.00, USD 85.00

When I was approached as a potential reviewer of this book I was immediately attracted by its title, and when it arrived by its presentation. It gave an instant impression of a high quality production, and indeed this was maintained throughout its length, ignoring the isolated superfluous apostrophes in "it's".

The book opens with the statement that it is not a textbook (a teaching text) but more of a workshop manual of statistical procedures. This is amply confirmed in the later chapters.

The first four chapters address general topics in statistical analysis, which are applicable to virtually any field, whilst revealing the particular (pharmacological) interests of the author, because multi-dimensional aspects of data-for example principal components - are introduced early in the text.
The treatment is necessarily brief, but I was slightly disappointed by the sparse use of small data sets that readers might try to test their understanding of the methods described. It turns out that experimental design in the classical sense would be difficult to apply in the many complex techniques that are presented later in the book. The scope of these techniques is amply illustrated by the impressive and helpful list of abbreviations and acronyms presented prior to the main text. It would be inappropriate to catalogue all these, but there are groups into which many techniques fall. Examples are unsupervised learning, in which cluster and factor analysis feature, and artificial neural networks. Such multivariate methods are presented and demonstrated as being well suited to the exacting environment of pharmaceutical research, where multiple dependent variables are as ubiquitous as multiple independents in some other fields. It should be carefully noted that anyone working in such areas will be wholly dependent on having access to complex software programs. Some techniques are dependent on heavily repeated analyses in which adjustable parameters are required in order to arrive at probability statements regarding outcomes. Rather oddly, amongst this welter of very advanced techniques and necessary software titles there are abbreviated tables of the $\mathrm{t}$ and $\mathrm{F}$ distributions. In the context of multiple regression these may be of interest, although any software will have such things as an integral part of the system. It is interesting that variable selection procedures are discussed in some detail and indeed seem to form a part of any (all?) learning procedures, but their impact on inferential statistics is not given much emphasis.

This book should provide those engaged in multidimensional experimentation a relatively compact (under 400 pages) oversight of the relative merits of numerous techniques, all of which are heavily computer dependent, and will be of especial interest to those working in the field of pharmaceutical research. It should also draw their attention to the roots of complex methods by means of its introductory chapters.

R. Edwards 